\title{
A DOR NA CRIANÇA COM CÂNCER: MODELOS DE AVALIAÇÃO*
}

Patrícia Torritesi**

Dulce Maria Silva Vendrúsculo***

TORRITESI, P.; VENDRÚSCULO, D.M.S. A dor na criança com câncer: modelos de avaliação. Rev.latinoam.enfermagem, Ribeirão Preto, v. 6, n. 4, p. 49-55, outubro 1998.

O controle e o alívio da dor na assistência à criança com câncer tem sido objeto de preocupação da enfermagem na busca de intervenções que possam minimizar ou evitar problemas de ordem fisico-emocional para estas crianças. A literatura da área médica, da psicologia e da enfermagem descrevem a dor através de aspectos fisiológicos, emocionais, comportamentais e ambientais, sendo divulgados vários modelos de escalas de avaliação e de controle da dor. Este estudo descreve alguns modelos de avaliação da dor em crianças e apresenta a adaptação do Modelo de Escala Analógica Visual de Faces de McGrath (1990), como instrumento a ser utilizado na assistência de enfermagem à criança com câncer. Embora a literatura utilizada neste estudo enfatize a avaliação e o controle da dor na criança com câncer, verificamos a viabilidade da aplicação deste modelo pela enfermagem em outras situações de dor.

UNITERMOS: avaliação da dor, criança, câncer

\section{INTRODUÇÃO}

A literatura nos últimos anos tem enfatizado a temática da dor em oncologia, focalizando os aspectos relacionados ao paciente adulto; entretanto o controle e o alívio da dor da criança com câncer são considerados significativos na assistência pediátrica. A maioria dos textos pediátricos não faz referências a respeito do controle da dor associado ao câncer infantil. Cumpre mencionar que a complexidade da pesquisa sobre a dor na infância esbarra, em geral em dificuldades de avaliação e de problemas éticos.

A Organização Mundial de Saúde vêm reconhecendo tais similaridades no controle da dor em adultos. Em resposta a esta questão, têm coordenado o desenvolvimento de várias iniciativas nacionais, enfatizando a inadequação do controle da dor em oncologia. Tais iniciativas tem como resultado o desenvolvimento de modelos para este controle, os quais, entretanto, não têm sido desenvolvidos para serem aplicados à criança ${ }^{7}$.

A conferência sobre o Controle da Dor no Câncer Infantil realizada nos Estados Unidos da América em 1988, teve como propósito consolidar alguns conhecimentos sobre esta temática, sendo prioritária a atuação dos vários setores de assistência, incluindo a enfermagem, a pediatria, a neurologia, a psicologia, a anestesiologia e a psiquiatria, com a finalidade de adequar uma metodologia de intervenção para dor do câncer e para a dor associada aos procedimentos diagnósticos ${ }^{7}$.

$\mathrm{O}$ controle e o alívio da dor na assistência à criança com câncer têm sido objeto de preocupação constante da equipe de enfermagem, na busca de intervenções que possam minimizar ou evitar problemas de ordem físico-emocional, relacionados ao tratamento, à evolução da doença e à assistência à criança em fase terminal.

A enfermagem atua inúmeras vezes com a criança com dor, percebendo assim que o aumento da ansiedade e do desconforto, compromete ainda mais o seu estado geral. Nesse sentido, a responsabilidade de promover o alívio da dor e o conforto do paciente exige uma precisa avaliação dos aspectos fisiológicos, emocionais, comportamentais e ambientais que desencadeiam ou exarcebam o quadro álgico na criança. $\mathrm{Na}$ assistência à criança em sua integralidade, devem

\footnotetext{
* Trabalho apresentado no $47^{\circ}$ Congresso Brasileiro de Enfermagem, $1^{\circ}$ lugar no prêmio Marina de Andrade Resende

** Aluna do Curso de Graduação em Enfermagem da Escola de Enfermagem de Ribeirão Preto-USP e bolsista de iniciação científica $-\mathrm{CNPq}$

*** Docente junto ao Departamento de Enfermagem Materno-Infantil e Saúde Pública da Escola de Enfermagem de Ribeirão PretoUSP, orientadora
} 
ser consideradas as situações de desconforto e de dor vivenciadas por esta, objetivando uma melhor qualidade de vida desses pacientes.

Resultante das considerações acima, o objetivo deste estudo foi revisar brevemente através da literatura, modelos de avaliação do quadro álgico na criança e propor instrumento a ser utilizado na avaliação do quadro álgico na criança com câncer.

\section{A PROBLEMÁTICA DA DOR NA CRIANÇA COM CÂNCER}

\section{Aspectos fisiológicos e psicológicos da dor}

A dor é uma sensação individual e muito pessoal do ser humano, que se manifesta mediante uma resposta fisiológica; é uma sensação, mas também um fenômeno emocional que leva a um comportamento de fuga e proteção; deve ser entendida como um fenômeno muito complexo, afetado por variações biológicas, intelectuais, emocionais e culturais ${ }^{1,3,5,7}$

A Associação Internacional para o Estudo da Dor define-a como uma desagradável sensação ou experiência emocional associada com dano tecidual real ou potencial, ou descrita em termos deste dano ${ }^{3}$.

A dor em oncologia se apresenta de modo agudo ou crônico. A dor aguda é breve, de fácil localização, caracterizada por pontadas, podendo provocar dilatação da pupila, sudorese, maior esforço cardíaco, fraqueza, dentre outros sintomas. Requer uma abordagem quase que puramente sintomática relacionada a um determinado transtorno físico; sendo que na maioria das vezes, o tratamento analgésico adequado e intervenções específicas levam ao desaparecimento do quadro álgico e de suas conseqüências. A dor crônica apresenta-se persistente, de difícil localização, causando distúrbio do sono, anorexia, diminuição da libido, desesperança e ansiedade ${ }^{3}$.

Segundo DELGADO ${ }^{3}$, a dor em oncologia tem características peculiares. Para estes pacientes a dor tende a ser contínua agravando-se na medida em que há evolução da doença neoplásica, levando o paciente 'a exaustão física e mental.

O quadro álgico está presente em 60 à $80 \%$ dos pacientes com tumores avançados, tornando-se mais complexo diante da refratariedade ao tratamento antineoplásico e das dificuldades de intervenções com medicações ou procedimentos analgésicos. Nestes casos, freqüentemente a dor é incapacitante.

Existem referências a métodos de controle primário da dor, como cirurgia, radioterapia e quimioterapia, nos quais a atuação é diretamente na doença neoplásica, e a de controle sistemático como analgésicos sistêmicos, de bloqueios e interferências nos mecanismos sensitivos e nervosos de condução da dor, controle da ansiedade, e depressão e, finalmente, a antibioticoterapia e os antiinflamatórios que atuam em situações específicas relacionadas ao quadro irreversível da doença ${ }^{3,7}$

A abordagem da dor inclui um outro conceito, o de pessoa, entendendo-se o indivíduo como o ser que possui bom senso, liberdade, sensibilidade, situado numa família. Nesta abordagem, o tratamento da dor deve ser realizado por uma equipe multiprofissional - o próprio paciente e família, as equipes médica e de enfermagem, psicólogos, assistentes sociais, religiosos e outros, que podem interferir no processo doloroso ${ }^{1,3}$.

A enfermagem pela própria característica de seu trabalho, defronta-se inúmeras vezes com situações dessa natureza, nas quais a dor compromete o estado geral do paciente, provocando aumento da ansiedade e do desconforto interferindo na qualidade do cuidado prestado. O quadro álgico reflete nas condições psicossociais dos familiares dificultando o relacionamento da equipe assistencial com a criança e sua família.

\section{Avaliação da dor}

Embora a preocupação com a existência da dor vivenciada pela criança, bem como a sua avaliação tenha sido objeto de várias pesquisas, na opinião de $\mathrm{CLARO}^{3}$, este tema ainda é pouco investigado e muitas vezes, subestimado por profissionais de saúde que convivem com a presença da dor como sintoma de inúmeras patologias. Entendemos que o limitado número de estudos da dor em crianças decorre das dificuldades relacionadas à própria definição da dor, sendo a mesma entendida como uma experiência sensorial e emocional desagradável, associada a um dano tecidual real ou potencial. Ainda outros aspectos apontados referem-se a dificuldade clínica da diferenciação da dor e ansiedade, e das características e intensidade da dor ${ }^{2,3}$

$\mathrm{Na}$ assistência à criança com dor algumas considerações importantes devem ser ressaltadas:

- a queixa de dor referida pela criança é o melhor indicador que deve ser avaliado;

- alterações do comportamento como choro, irritabilidade, isolamento social, distúrbios do sono e da alimentação são indicativos de um quadro álgico;

- recém nascidos e crianças menores não são menos sensíveis aos estímulos dolorosos do que crianças mais velhas e adultos. Nestes casos a observação das mudanças comportamentais e de variações fisiológicas (freqüência cardíaca, freqüência respiratória, e pressão arterial) são indicadores potenciais da dor sentida;

- a intensidade da dor está relacionada a outras causas indeterminadas que devem ser pesquisadas ${ }^{5,7}$. 
Para a enfermagem a avaliação da dor em crianças é um fator relevante na assistência, uma vez que cabe a esses profissionais a tomada de decisões sobre medidas de alívio da dor e do desconforto do paciente. A ausência de um processo que propicie uma avaliação mais adequada do quadro álgico na criança leva, muitas vezes, a não-identificação e ao controle inadequado da dor por parte da equipe médica e de enfermagem.

A avaliação compreensiva da dor deve incluir mais do que medidas de intensidade ou severidade desta, sendo propostos na literatura vários métodos para esta avaliação ${ }^{5}$.

O desenvolvimento da criança pode determinar o método de abordagem de avaliação da dor a serem utilizados. Até os três anos de idade esta avaliação tem como critérios as respostas comportamentais e as fisiológicas; a partir desta idade, pode ser utilizado o relato da criança a respeito de suas próprias experiências para avaliar a intensidade ou severidade do quadro álgico, sendo possível a utilização de instrumentos apropriados para avaliação da dor.

$\mathrm{Na}$ seleção do instrumento deve-se levar em consideração as condições da criança, idade, sexo, aspectos sócio-culturais e desenvolvimento cognitivo. A seguir discorreremos sobre os modelos de avaliação.

\section{MODELOS DE AVALIAÇÃO}

Os estudos sobre avaliação do comportamento têm sido desenvolvidos na área da psicologia comportamental contribuindo assim na avaliação de respostas à dor, sobretudo no cuidado a recém-nascidos e a lactentes, e às crianças e adultos em situações muito estressantes e dolorosas.

\section{Avaliação da informação da criança}

A informação do paciente pode ser obtida através de comunicação verbal e não verbal: podem ser utilizadas listas de palavras em forma de "check-list", o uso de brinquedos e desenhos para qualificar suas experiências de dor. A resposta verbal pode ser expontânea ou solicitada e pode referir aspectos sensoriais, emocionais ou cognitivos para caracterizar a experiência dolorosa. Crianças acima de 7 anos são capazes de detalhar melhor suas experiências dolorosas e suas necessidades de conforto e alívio.

WILSON et al. ${ }^{8}$ faz referência a um instrumento clássico para avaliar a dor - o Questionário da Dor desenvolvido por MELZACK ${ }^{6}$, o qual tem sido largamente utilizado em pesquisas e na clínica. Os dados obtidos através deste questionário permitem identificar a idade, o diagnóstico, e terapia analgésica, através de parâmetros sensoriais, afetivos, avaliativos e mistos.

\section{Avaliação comportamental}

As manifestações subjetivas da dor podem ser observadas através de respostas comportamentais como expressão facial, postura e verbalização ${ }^{5,7}$.

Algumas escalas para avaliação comportamental de crianças com dor têm sido descritas a partir de observações realizadas no período pós operatório ou durante procedimentos de injeções. Nestas observações são utilizados escores obtidos através de observação de comportamentos por meio de um "check-list" contendo alguns indicadores da dor conforme o exemplo a seguir (Quadro 1).

Quadro 1 - Indicadores comportamentais da dorcheck-list

\begin{tabular}{|c|l|l|}
\hline COMPORTAMENTo & AUSENTE & PRESENTE \\
\hline *Irritabilidade & & \\
\hline *Isolamento Social & & \\
\hline *Choro & & \\
\hline *Distúrbio de Sono & & \\
\hline *Expressão facial & & \\
\hline *Redução do apetite & & \\
\hline *Redução do lazer & & \\
\hline *Redução da atenção & & \\
\hline
\end{tabular}

Fonte: SCHECHTER ${ }^{7}$

\section{Avaliação da intensidade da dor}

Alguns instrumentos de avaliação da intensidade da dor são aplicados à crianças de 3 a 7 anos, cuja etapa de desenvolvimento dificulta a capacidade de abstração, simbolização e quantificação, sendo desta forma a avaliação da dor um desafio. A seguir descreveremos esses instrumentos:

- Modelo de Esquema Corporal - visa descrever a própria dor, sua natureza e localização. A criança é orientada a indicar em um desenho de corpo humano o local da dor sentida ${ }^{8}$.

- Escala de OUCHER - dispõe de seis fotografias de crianças chorando apresentando diferentes níveis de expressões faciais de desconforto. A criança relaciona a expressão que melhor reflete sua experiência de dor ${ }^{7}$.

- Escala de Cores - o paciente é orientado a escolher uma das três cores que considera melhor descrever a 
intensidade da dor Este modelo permite que, a criança utilize mais a sua intuição que a avaliação cognitiva. Uma outra variação desta escala de cores permite que a criança localize sua dor em um esquema corporal e posteriormente, colorir o local com a cor que mais expresse sua dor ${ }^{4}$.

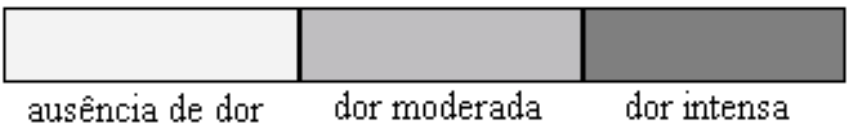

Figura 1 - Escala de cores

Fonte: LAVIGNE(1986)

- Escala Linear Analógica Visual - indicada por uma linha reta, com extremidades significando de um lado, ausência de dor, do lado oposto, a maior intensidade de dor já sentida pela criança, a qual localiza-a no contínuo da escala, o grau da intensidade de sua dor ${ }^{7}$.

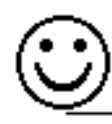

ausência de dor

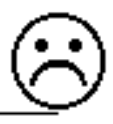

dor severa
Figura 2 - Escala linear analógica visual

Fonte: SCHECHTER(1990)
- Escala Linear Analógica Não Visual - constitui-se em outra variante da escala apresentada anteriormente, na qual é feita a quantificação da intensidade dolorosa através de escores que variam de zero a dez, sendo essa caracterizada por dor leve, intensa, aguda ou muito intensa ${ }^{7}$.

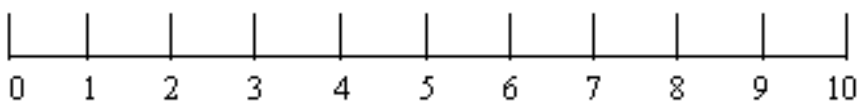

ausência de dor

dor severa

\section{Figura 3 - Escala linear analógica não visual}

Fonte: SCHECHTER(1990)

- Escala Analógica Visual de Faces - proposta por McGRATH $^{5}$, constituída por expressões faciais em cada extremidade de uma linha horizontal, as quais demonstram variação de amplitude de ausência de dor até dor intensa - Escala de Faces - adaptada por CLARO ${ }^{2}$, que mostrou figuras desenhadas por Maurício de Sousa*, representando os personagens criados por ele, Cebolinha e Mônica com diferentes expressões faciais. A escala é composta por cinco expressões, as quais variam da expressão sem dor até a dor insuportável, sendo $0=$ sem dor, $1=$ dor leve, $2=$ dor moderada, $3=$ dor forte, $4=$ dor insuportável.
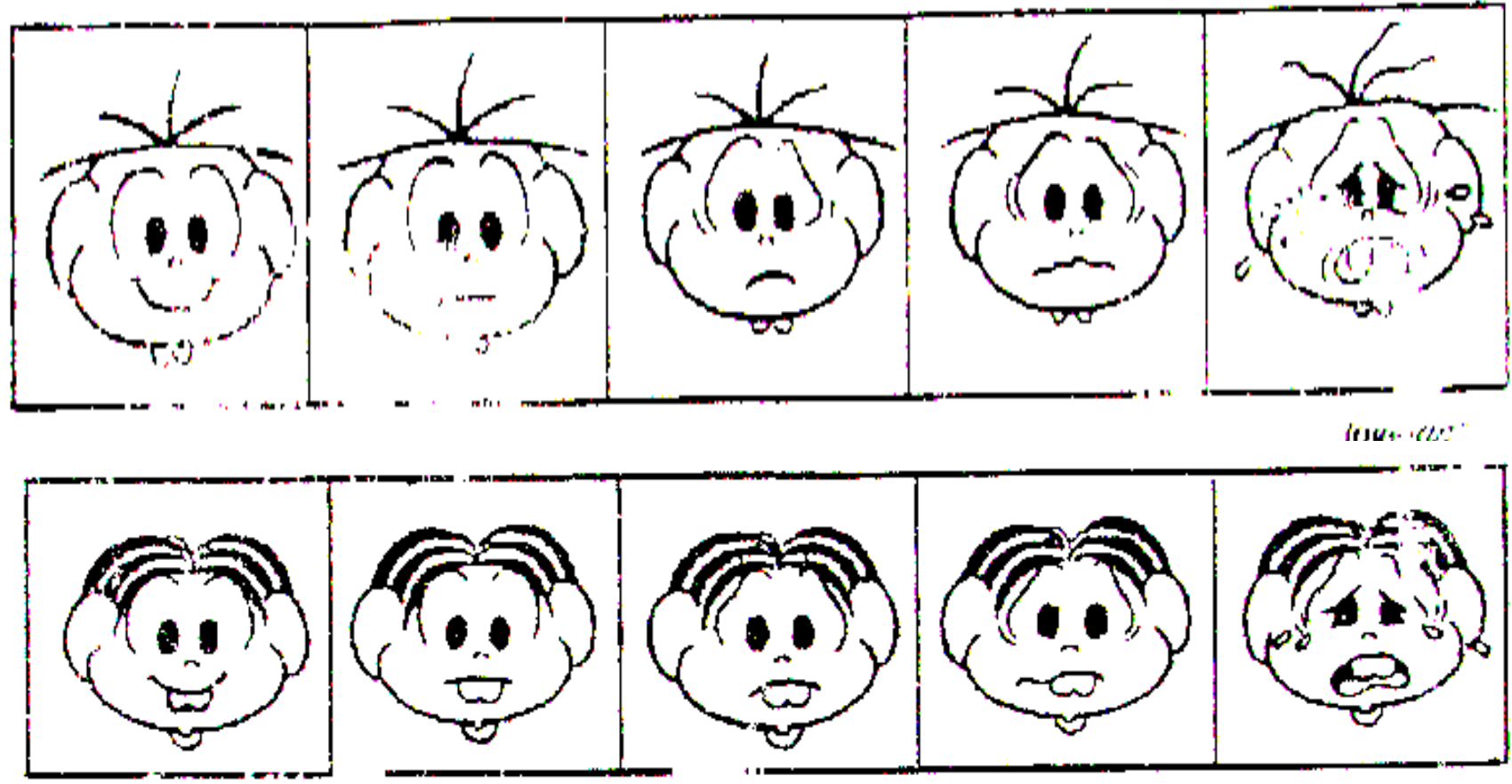

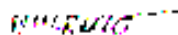

Figura 5 - Escala de faces (diferentes expressões do cebolinha aplicadas para os meninos e da mônica para as meninas)

Fonte: CLARO (1993)

**** Maurício de Sousa - jornalista, desenhista, autor de histórias em quadrinhos e presidente da Editora Maurício de Sousa 
Após essa breve revisão da literatura entendemos a necessidade da avaliação efetiva da dor clínica a ser feita pelos enfermeiros. Para tal, apresentaremos a seguir o Modelo de Escala Analógica Visual de faces de McGrath, 1990, como sugestão de instrumento a ser utilizado.

\section{MODELO DA ESCALA ANALÓGICA VISUAL DE FACES (McGRATH,1990)}

A literatura demonstra que a avaliação da dor efetuada pela enfermagem, na maioria das vezes não contribui para um efetivo controle do quadro álgico, o conforto da criança e a prescrição analgésica, interferindo assim na qualidade da assistência prestada. Muitas vezes, quando prescrição analgésica é indicada como "se necessário", deparamos com a ausência de parâmetros de avaliação comportamental ou de intensidade da dor, impedindo assim a tomada de decisão eficaz e a possibilidade de desenvolvimento de mais conforto e bem estar para essas crianças ${ }^{3}$

Após a descrição dos vários modelos utilizados na avaliação do quadro álgico da criança, propusemonos a apresentar o modelo da Escala Analógica Visual de Faces de McGRATH ${ }^{5}$ como instrumento para orientação da assistência de enfermagem, focalizando neste estudo a avaliação da dor na criança com câncer. De acordo com as informações referidas na literatura, este modelo melhor se adapta para crianças com maior capacidade de cognição e abstração.

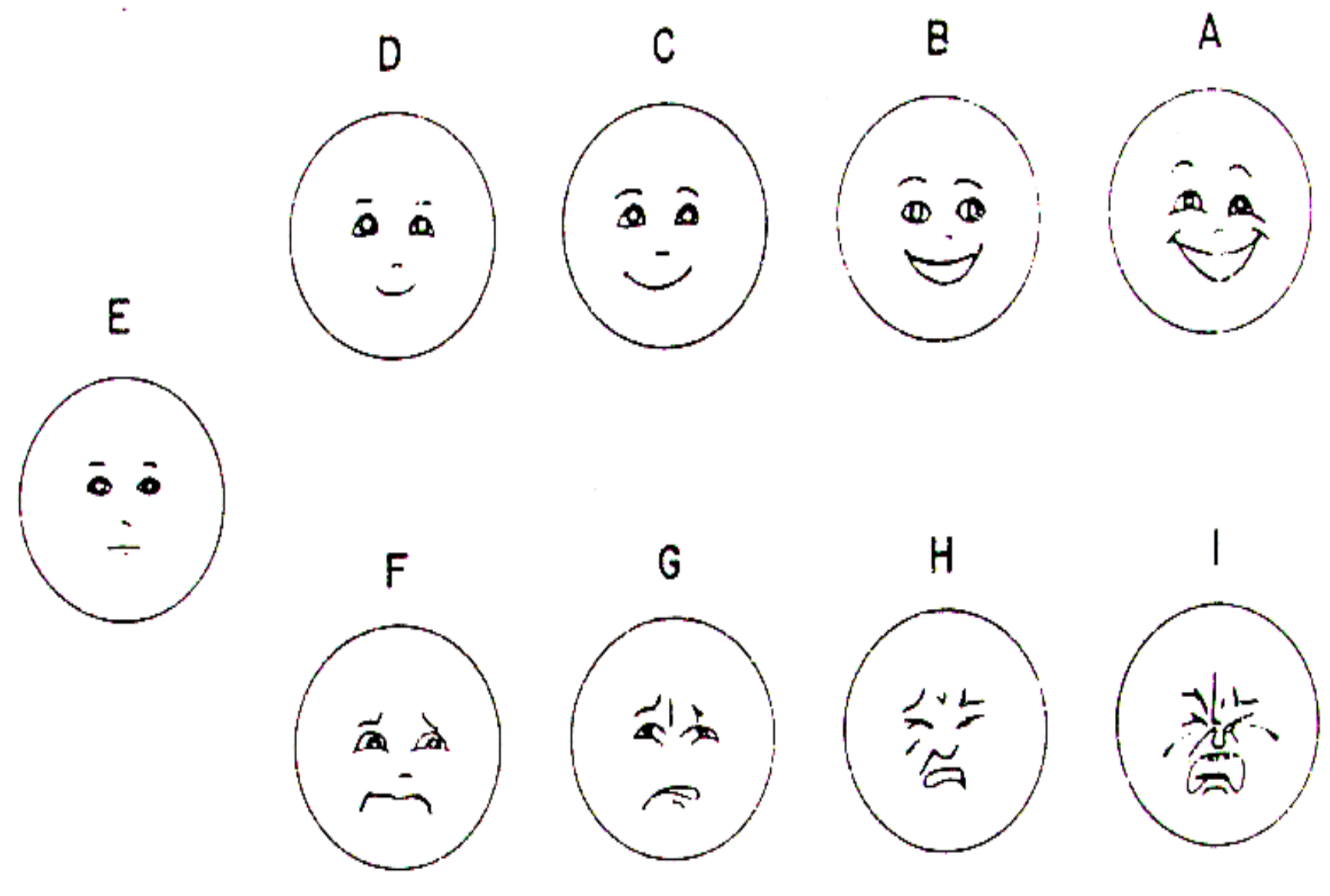

Figura 5 - Escala analógica visual de faces

Fonte: McGRATH (1990)

Neste modelo a representação da Escala de Faces apresenta dois pólos de significados distintos, correspondendo a uma amplitude de variações de efeitos positivos e negativos em relação à dor.

- As Figuras de A à $\mathbf{D}$ representam variações de estímulos positivos, caracterizando que a criança não sente dor;

- A Figura $\mathbf{E}$ é o ponto neutro da escala;

- As Figuras de F à I vão representar uma amplitude de variação negativa referente a diferentes graus de intensidade da dor;

- A Figura $\mathbf{F}$ indica que a dor está presente, mas poderá ser facilmente ignorada pela criança. Neste caso a presença dos familiares, um ambiente favorável, medidas de conforto, segurança e compreensão desempenhadas pelo profissional de enfermagem podem favorecer uma melhor adaptação da criança a seu estado físico e emocional; 
- A Figura $\mathbf{G}$ indica dor presente, com uma certa intensidade, que não pode ser ignorada pela criança, mas que ainda não interfere no seu comportamento. A verbalização da criança é um indicador para esta avaliação que deverá ser precedida de medidas para o alívio da dor e conforto da criança;

- A Figura $\mathbf{H}$ indica dor presente, não pode ser ignorada pela criança, interferindo no seu comportamento. $\mathrm{O}$ resultado da observação das respostas comportamentais da criança pode indicar necessidades de intervenção além daquelas propiciadas pela enfermagem, como o uso de analgésicos;

- A Figura I indica dor presente, em maior grau de intensidade, requerendo uma maior intervenção da equipe, devendo ser observados os aspectos físicos e psicológicos com intervenções específicas e técnicas analgésicas apropriadas.

O interesse pela utilização deste modelo é na tentativa de facilitar a avaliação do quadro álgico da criança, durante procedimentos terapêuticos invasivos como a cirurgia, a quimioterapia, a punção intra-tecal e medular.

\section{ALGUMAS CONSIDERAÇÕES FINAIS}

Para a criança com câncer, a dor e o desconforto determinam um quadro de ansiedade comprometendo os aspectos físicos, emocionais e comportamentais, prejudicando o seu estado geral ou exacerbando o quadro álgico. Frente a essas colocações, reforça-se a questão orientadora desse estudo: COMO AVALIAR OQUADRO ÁLGICO DA CRIANÇA COM CANNCER?

Diante dessa responsabilidade, surge a necessidade de avaliar mais objetiva e precisamente o quadro álgico, propiciando uma melhor qualidade na assistência de enfermagem e um adequado tratamento da dor. A utilização do modelo da Escala de McGrath para o controle e avaliação da dor em crianças com câncer tem como objetivo contribuir para uma assistência de enfermagem voltada para a sua integralidade física, emocional e social. O uso deste instrumento de avaliação poderá orientar a assistência de enfermagem à criança com dor, decorrente de outros processos patológicos, cirúrgicos e traumáticos que venham comprometer a integridade física e psicológica das crianças, contribuindo assim para uma melhor qualidade de assistência.

\section{PAIN IN CHILDREN WITH CANCER: EVALUATION MODELS}

Control and alleviation of pain in the care to children with cancer are objects of nursing concern in the search for interventions that are able to minimize or avoid physical-emotional problems in these children. Medical, psychological and nursing literatures describe pain through physiological, emotional, behavioural and environmental aspects in several models of scales of pain evaluation and control. This study describes some models of evaluation of pain in children and presents the adaptation of the Scale Model of Visual Analogy of Faces by McGrath (1990), as an instrument to be used in nursing care to children with cancer. Although the literature utilized on this study gives emphasis on the evaluation and control of pain in children with cancer, we verify the viability of applying this model by nursing in other situations of pain.

KEY WORDS: evaluation of pain, child, cancer

\section{EL DOLOR EN NIÑOS CON CÁNCER: MODELOS DE EVALUACIÓN}

El control y alivio del dolor en la asistencia a niños con cáncer ha sido objeto de inquietud de la enfermería en busca de intervenciones que puedan minimizar o evitar problemas de orden físico-emocional para estos niños. La literatura de las áreas médica, psicología y enfermería describen el dolor a través de aspectos fisiológicos, emocionales, comportamentales y ambientales, siendo divulgados vários modelos de escalas de evaluación y control del dolor. Este estudio describe algunos modelos de evaluación del dolor en niños y presenta la adaptación del Modelo de Escala Analógica Visual de Facetas de McGrath (1990), como instrumento a ser utilizado en la asistencia de enfermería a niños con cáncer. Sin embargo la literatura utilizada en este estudio enfatice la avaluación y el control de el dolor en el niño con cáncer, verificamos la viabilidad de la aplicación de este modelo por el profesional de enfermería en otras situaciones del dolor.

TÉRMINOS CLAVES: evaluación del dolor, niño, cáncer

\section{REFERÊNCIAS BIBLIOGRÁFICAS}

01. CASTILHO, E.E. El dolor en los ninõs. Rev. Educ Enfermeria, Medelin, v.2, p.41-40, septiembre 1987.
02. CLARO, M.T. Escala de faces para avaliação da dor em crianças: etapa preliminar. Ribeirão Preto, 1993. p. 1-50. Dissertação (Mestrado)Escola de Enfermagem de Ribeirão Preto, Universidade de São Paulo. 
03. DELGADO, G.L. A dor em oncologia. São Paulo: Atheneu/EDUSP, 1988.

04. LAVIGNE, J.V. et al. Psychological aspects of painful medical condictions in children. Pain, v.27,p.133146, 1986.

05. McGRATH, P.J. et al. Report of the Subcommithes on assement and methodologic issues in the management of pain in childhood cancer. Pediatrics, v.86, n.5, p.814-817, November, 1990.
06. MELZACK, R. The Mc Gill pain questionnaire: major properties and scoring methods. Pain, v.1, p.277299, 1975.

07. SCHECHTER, N.L. et al. Report of the Consensus Conference on the Management of pain in childhood cancer. Pediatrics, v.86, n.5 ,p.818-834, November, 1990.

08. WILSON, J.; BARNETE; LYNN,B. A research base for nursing care. London: Scrittori Press London, 1991.p.118-127. 